\title{
Internalized stigma is associated with psychological distress among patients with substance use disorders in Egypt
}

\author{
Amira Mohammed Ali ${ }^{1,2 *}$ \\ ${ }^{1}$ Department of Mental Disorder Research, National Institute of Neuroscience, National Center of Neurology and Psychiatry, Tokyo, Japan \\ ${ }^{2}$ Department of Psychiatric Nursing and Mental Health, Faculty of Nursing, Alexandria University, Alexandria, Egypt
}

\begin{abstract}
Aim: This study aimed to investigate stigma, psychological distress, and their correlates among Egyptian substance users.

Introduction: Although stigma of substance abuse and its impact on psychological health have recently received considerable attention, these problems are underaddressed among Arab patients.

Methods: This cross-sectional study involved a convenient sample of 149 inpatient substance users (142 males, mean age=32.5 years, SD=6.8 years, range: $19-60$ years) They completed the Self-Stigma in Alcohol Dependence Scale and the Depression Anxiety Stress Scale-21 Hierarchical multiple regression was conducted to identify correlates of stigma and psychological distress.

Results: In adjusted analysis, young age, abuse of alcohol and Bango significantly predicted stigma Shame and shorter hospital stays were associated with higher psychological distress, explaining $31 \%$ of the variance, and bait along with stereotype agreement explained $36 \%$ of the variance in depression scores.

Discussion: Younger substance users are more likely to internalize stigma Meanwhile, the shame component of internalized stigma has the worst effect on psychological health.

Implications for practice: The findings necessitate stigma interventions that target shame reduction to enhance the psychological well-being and recovery of substance users.
\end{abstract}

Abbreviations: DASS-21: Depression Anxiety Stress Scale-21; PTSDs: Post-traumatic stress disorders; SSAD: Self-Stigma in Alcohol Dependence Scale; SUDs: Substance use disorders.

\section{Aim}

This study aimed to examine self-stigma, psychological distress, and their correlates among Egyptian substance users This study contributes to the literature since, to date, there is neither measures nor published studies of self-stigma of abuse of illicit substances and related psychological distress among Arabs.

\section{Hypotheses}

Taking the above theoretical background in mind, the researcher expected to find a strong association between self-stigma and abuse of certain substances as well as sociodemographic variables Additionally, this study hypothesized that perceived and internalized stigmas would be strong predictors of psychological distress Likewise, it was expected that chronicity of SUDs, frequent relapse, cannabis and heroin use, employment and housing problems would be predictors of higher psychological distress scores.

\section{Introduction}

Worldwide, over 29 million people suffer from substance use disorders (SUDs) [1]: problematic use of alcohol or other substances that cause clinically significant impairment and noticeable distress [2] These disorders represent a challenge for Arab countries, which witnessed an outburst of illicit drugs in the last two decades [3] Egypt reports SUDs among $12.4 \%$ of the population, which is more than twice the global rate (5\%) [4] The problem peaks in young groups and workers in manufacturing industries Concerns have been raised since most cheap and accessible substances have poor quality and lead to severe cognitive and behavioral impairments e.g Bango and tramadol; co-morbidity of multiple SUDs is common $[5,6]$ SUDs patients contract serious infections, suffer from poor physical and psychological health, social problems, criminality, and are at a high risk of premature death [1] In addition, they face a widespread stigma because use of illicit substances is considered a deviant social status [7].

Stigma is a social process: the public blame substance users for their problems and stereotype them as weak-willed, violent, and dangerous $[8,9]$ The public set a status hierarchy, which has drug users at the bottom; they exclude, reject, discriminate against, and unfairly treat substance-using individuals $[10,11]$ Thus, the label of a "drug

*Correspondence to: Amira Mohammed Ali, Department of Mental Disorder Research, National Institute of Neuroscience, National Center of Neurology and Psychiatry, 4-1-1, Ogawahigashi, Kodaira, Tokyo 187-8502, Japan, E-mail: mercy.ofheaven2000@gmail.com

Key words: anxiety, egypt, depression, psychological distress, self-stigma, shame, substance-related disorders

Received: October 17, 2019; Accepted: October 30, 2019; Published: November 04, 2019 
user" seriously threatens individuals' survival-being denied basic life opportunities for proper work/income, housing, education, health care/insurance services, social interactions and networks [12,13] Drug related discrimination is the worst stigma reported by people with multiple stigmata [10].

From the perspective of the stigmatized person, stigma is a selfdevaluation process, which is composed of 4 inter-related stages: 1) awareness of the negative attitudes held by the public toward substance users e.g they say that drug users are untrustworthy; 2) personal agreement with public opinions e.g I think that drug users are untrustworthy; 3) self-occurrence and application of stereotypes to self (in patients who identify with a stigmatized group and view stereotypes as legitimate) e.g I am untrustworthy because I use drugs; 4) shame and demoralization-flawed self-views cause loss of self-esteem and self-efficacy The first two stages embrace none self-relevant stigma perceptions (perceived stigma) while the last two stages constitute internalized stigma $[14,15]$

Stigma negatively affects both physical and psychological health Patients with high stigma share used syringes; engage in self-harm, risky sexual behaviors, and criminal activities $[16,17]$ Further, stigma impedes recovery and help seeking behaviors Despite the availability of effective therapies for SUDs, only 1 out 6 patients receive treatment [1], and most treatment seekers drop out before treatment completion [18] Reluctance to use health services is associated with higher stigma perceptions [19], fear of having legal problems, or fear of rejection by care providers [7] There is evidence that substance users are rejected by professionals in treatment facilities $[17,20,21]$.

Symptoms of depression and anxiety, herein referred to as psychological distress, are wide-spread among substance users [22] On one hand, depression, anxiety, and post-traumatic stress disorders (PTSDs) are highly co-morbid with SUDs-they are encountered by up to $3 / 4$ of the patients $[2,23,24]$ On the other hand, these symptoms may stem from the negative consequences of stigma and discrimination (e.g lack of employment and housing), which represent a frequent source of stress for SUDs patients $[7,21,25]$.

Chronic stress leads to depletion of internal resources, inability to predict or regulate one's emotions, and development of feelings of shame [26] (the deepest component of stigma) Most SUDs patients inhibit negative emotions, which in turn stimulates frequent rumination and intrusions i.e., the thought process spins repeatedly around defeating self-views, which further impedes the ability of problem solving and goal achievement [27,28] As a result, people develop a sense of powerlessness, low self-esteem and self-efficacy, poor social functioning, poor quality of life, distress, and depressive symptoms [29].

Fear of acquiring the official label (by contacting mental health services) drives SUDs patients to keep away from treatment settings and to manage their emotional problems on their own [17] Nonetheless, SUDs patients have limited ability to withstand negative emotions [30]; they find illicit drugs an appealing method to control their symptoms of depression and anxiety [31] Nevertheless, intoxication worsens the depressive symptoms, and persons get into a vicious circle where they re-use illicit substances to heal their endless misery Therefore, psychological distress leads to continued abuse, treatment failure, and relapse [24,32].

\section{Materials and methods}

Study design, participants, and procedure: This cross-sectional study was conducted at a government psychiatric hospital in
Alexandria between January 2014 and May 2015 Patients were eligible if they were literate, free from severe mental disorders, and willing to sign an informed consent Records were checked to identify eligible participants For all interested participants, the study details were explained, anonymity was ensured, and informed consent was obtained Of inpatients $(N=420), 51.2 \%$ were eligible to participate; however, only $35.5 \%$ took part in the study This study included a convenient sample of 149 detoxified SUDs inpatients Because of lack of a private place, questionnaires were handed to participants either in the lounge or the visit room The researcher was available for any clarifications The study was approved by the University of Alexandria Committee for Research Ethics (July 2011).

\section{Measurements}

Self-Stigma in alcohol dependence Scale (SSAD): The SSAD was used to measure stigma It consists of 4 subscales which operationalize stages of internalizing stigma indicated beforehand (awareness, agreement, self-occurrence, and shame) Each subscale has 16 items All items convey negative views of SUDs patients e.g unreliable, dirty, and less intelligent Responses were on a 5-point scale (1=strongly disagree and $5=$ strongly agree) Higher scores indicate higher stigma (Schomerus et al., 2011) The SSAD was translated into Arabic Because population of the current study used alcohol and other drugs, the scale was modified by substituting 'alcohol dependence' with 'substance dependence' Alpha coefficients for the SSAD subscales in this study were adequate $(.81, .86, .83, .84)$.

The depression anxiety stress scale-21 (DASS-21): The DASS21 has 21 items in 3 subscales of 7 items each They assess depressive symptoms (e.g feeling down-hearted and blue), anxiety symptoms (e.g feeling close to panic), and general stress symptoms (e.g having a tendency to over-react to situations) Responses were on a 4-point scale $(0=$ did not apply to me at all and $3=$ applied to me most of the time) Higher scores indicate more psychological distress [33] The reliability of this scale was high (coefficient alpha $=.88$ ) [22].

Sociodemographics and clinical characteristics: This comprised 14 questions about gender, age, education, employment and housing status, substances used, long-life duration of use; along with relapse which was indirectly assessed through the number of previous quitting trials and hospital admissions; an open ended question was used to assess previous relapse factors.

Statistical analysis: All analyses were performed using IBM SPSS version 22 Continuous variables were checked for normality of distribution, and $\log 10$ function was used to transform skewed variables-all variables except self-occurrence, shame, and stress The researcher used recommended cut-off points of subscales of the DASS-21 [34] to create 3 categorical variables of depression, anxiety, and stress: scores above 14,10, and 19 were coded moderate/high and scores below the cut-off points were coded low Chi square test detected participants' demographic and clinical differences in both groups Then, mean items scores of the SSAD were calculated; a one sample t-test was conducted on the scores to evaluate whether their differences from a 'neutral score $=3$ 'on the SSAD were significant Pearson correlation coefficients assessed the association of stigma with disease chronicity, abused substances, and the demographic characteristics Pearson correlation coefficients also assessed the association of psychological distress, depression, anxiety, and stress with stigma, chronicity, relapse, and the demographic characteristics After assumptions testing, eight hierarchical multiple regression models adjusted for age and gender were conducted to predict stereotype awareness, agreement, self- 
occurrence, shame, psychological distress, depression, anxiety, and stress-the models included only outcomes with significant correlations in crude analysis (Tables 1-3) This was because variables were entered simultaneously in the models, and it was possible that the co-existence of many independent variables can reduce the predictive capability of predictors highly correlated (on their own) with outcomes in a linear regression.

\section{Results}

The sample comprised 149 participants ( 142 males, mean age $=32.5$ years, $\mathrm{SD}=6.8$ years, range: $19-60$ years) Half the participants were single (49.7\%), 76.5\% had high school or less, $26.2 \%$ were unemployed, $29.5 \%$ had insufficient income, and $81.9 \%$ were staying with their families Participants varied in their disease chronicity, previous hospital admission and quitting history; a range of abused substances and relapse factors was reported (Table 1).

Table 1 indicates moderate to severe depression, anxiety, and stress in $23.5 \%, 33 \%$, and $2 \%$ of the sample, respectively Female gender was associated with both depressive and anxiety symptoms Unemployment was associated with depressive symptoms Cannabis was significantly associated with anxiety while use of synthetic drugs was marginally associated with depressive symptoms Relapse due to stressors was associated with depressive symptoms whereas availability of money as a relapse factor was associated with anxiety symptoms.

Table 2 shows means, standard deviations, and correlations of the study variables The mean item scores (supplement) of the SSAD awareness and agreement were $4.01 \quad(S D=0.54)$ and 3.85 $(S D=0.63)$ - which significantly corresponded to the "agree" response ( $p$ values $=0.000$ ) Meanwhile, the mean item scores of the SSAD selfoccurrence and shame were (significantly below 3$) 2.75(S D=0.69)$ and $2.56(S D=0.73)$ respectively, $(p$ values $=0.000)$.

As shown in Table 2, 4 and 5 no associations were revealed between any of the stigma constructs and the sociodemographic characteristics except for age; however, stigma was correlated with use of alcohol and Bango, the number of abused drugs, disease chronicity, and the number of hospital admissions Both psychological distress and depression were positively correlated with stereotype self-occurrence and shame, and negatively correlated with chronicity, length of hospital

Table 1. Participants' sociodemographic characteristics, abused drug, and relapse factors N (\%)

\begin{tabular}{|c|c|c|c|c|c|c|c|c|c|c|}
\hline \multirow[b]{2}{*}{ Variables } & \multicolumn{2}{|c|}{ Depression } & \multirow[b]{2}{*}{$\mathbf{P}$} & \multicolumn{2}{|c|}{ Anxiety } & \multirow[b]{2}{*}{$\mathbf{P}$} & \multicolumn{2}{|c|}{ Stress } & \multirow[b]{2}{*}{$\mathbf{P}$} & \multirow{2}{*}{$\begin{array}{c}\text { Total }(\mathrm{N}=149) \\
\text { N }(\%)\end{array}$} \\
\hline & $\begin{array}{l}14<\text { Low } \\
(\mathrm{N}=113)\end{array}$ & $\begin{array}{c}14 \geq \text { High } \\
(\mathrm{N}=35)\end{array}$ & & $\begin{array}{c}10<\text { Low } \\
(\mathrm{N}=99)\end{array}$ & $\begin{aligned} & 10 \geq \text { High } \\
&(\mathrm{N}=49)\end{aligned}$ & & $\begin{array}{l}19<\text { Low } \\
(N=145)\end{array}$ & $\begin{array}{c}19 \geq \text { High } \\
(\mathrm{N}=3)\end{array}$ & & \\
\hline $\begin{array}{l}\text { 1.Gender } \\
\text { Males } \\
\text { Females }\end{array}$ & $\begin{array}{c}111(78.7) \\
2(28.6)\end{array}$ & $\begin{array}{l}30(21.3) \\
5(71.4)\end{array}$ & $.008^{* *}$ & $\begin{array}{c}98(69.5) \\
1(14.3)\end{array}$ & $\begin{array}{c}43(30.5) \\
6(85.7)\end{array}$ & $.006 * *$ & $\begin{array}{c}138(97.9) \\
7(100)\end{array}$ & $\begin{array}{c}3(2.1) \\
0(0)\end{array}$ & .864 & $\begin{array}{c}142(95.3) \\
7(4.7)\end{array}$ \\
\hline $\begin{array}{c}\text { 2. Education } \\
\text { High school or less } \\
\text { Some college or degree }\end{array}$ & $\begin{array}{l}87(76.3) \\
26(76.5)\end{array}$ & $\begin{array}{c}27(23.7) \\
8(23.5)\end{array}$ & .592 & $\begin{array}{l}75(66.4) \\
24(68.6)\end{array}$ & $\begin{array}{c}38(33.6) \\
11(31.4)\end{array}$ & .491 & $\begin{array}{c}112(99.1) \\
33(94.3)\end{array}$ & $\begin{array}{l}1(0.9) \\
\quad 2(5.7)\end{array}$ & .139 & $\begin{array}{l}114(76.5) \\
35(23.5)\end{array}$ \\
\hline $\begin{array}{l}\text { 3. Marital status } \\
\text { Single } \\
\text { Married } \\
\text { Divorced/widow }\end{array}$ & $\begin{array}{c}57(77) \\
41(74.5) \\
15(78.94)\end{array}$ & $\begin{array}{c}17(23) \\
14(25.5) \\
4(21.05)\end{array}$ & .930 & $\begin{array}{l}48(65.8) \\
40(72.7) \\
11(55)\end{array}$ & $\begin{array}{c}25(34.2) \\
15(27.3) \\
9(45)\end{array}$ & .308 & $\begin{array}{c}72(98.6) \\
54(98.2) \\
19(95)\end{array}$ & $\begin{array}{c}1(1.4) \\
1(1.8) \\
1(5)\end{array}$ & .755 & $\begin{array}{l}74(49.7) \\
55(36.9) \\
20(13.5)\end{array}$ \\
\hline $\begin{array}{l}\text { 4. Employment } \\
\text { Employed } \\
\text { Unemployed }\end{array}$ & $\begin{array}{l}89(80.9) \\
24(63.2)\end{array}$ & $\begin{array}{l}21(19.1) \\
14(36.8)\end{array}$ & $.025^{*}$ & $\begin{array}{l}77(70.6) \\
22(56.4)\end{array}$ & $\begin{array}{c}32(29.4) \\
17(43.6)\end{array}$ & .079 & $\begin{array}{l}107(98.2) \\
38(97.4)\end{array}$ & $\begin{array}{l}2(1.8) \\
1(2.6)\end{array}$ & .603 & $\begin{array}{c}110(73.8) \\
39(26.2)\end{array}$ \\
\hline $\begin{array}{c}\text { 5. Income } \\
\text { Not enough } \\
\text { Enough } \\
\text { More than enough }\end{array}$ & $\begin{array}{c}31(72.1) \\
76(78.4) \\
5(71.4)\end{array}$ & $\begin{array}{l}12(27.9) \\
21(21.6) \\
2(28.6)\end{array}$ & .692 & $\begin{array}{c}29(65.9) \\
64(66.7) \\
6(85.7)\end{array}$ & $\begin{array}{c}15(34.1) \\
32(33.3) \\
1(14.3)\end{array}$ & .443 & $\begin{array}{r}44(100) \\
93(96.9) \\
7(100) \\
\end{array}$ & $\begin{array}{l}0(0) \\
3(3.1) \\
0(0)\end{array}$ & .197 & $\begin{array}{c}44(29.5) \\
97(65.1) \\
7(4.7)\end{array}$ \\
\hline $\begin{array}{l}\text { 6. Housing status } \\
\text { With family } \\
\text { Alone } \\
\text { With relatives }\end{array}$ & $\begin{array}{c}95(77.9) \\
14(77.8) \\
4(50)\end{array}$ & $\begin{array}{c}27(22.1) \\
4(22.2) \\
4(50)\end{array}$ & .197 & $\begin{array}{c}83(68.6) \\
11(61.1) \\
5(55.6)\end{array}$ & $\begin{array}{l}38(31.4) \\
7(38.9) \\
4(44.4)\end{array}$ & .621 & $\begin{array}{l}119(98.3) \\
18(100) \\
8(88.9)\end{array}$ & $\begin{array}{c}2(1.7) \\
0(0) \\
1(11.1)\end{array}$ & .123 & $\begin{array}{c}122(81.9) \\
18(12.1) \\
9(6)\end{array}$ \\
\hline $\begin{array}{l}\text { 7. Abused drugs } \\
\text { Cannabis } \\
\text { Bango } \\
\text { Heroin } \\
\text { Synthetic drugs } \\
\text { Alcohol } \\
\text { Other drugs }\end{array}$ & $\begin{array}{l}87(78.4) \\
31(77.5) \\
91(76.5) \\
93(79.5) \\
48(77.4) \\
23(74.2)\end{array}$ & $\begin{array}{c}24(21.6) \\
9(22.5) \\
28(23.5) \\
24(20.5) \\
14(22.6) \\
8(25.8)\end{array}$ & $\begin{array}{l}.215 \\
.514 \\
.559 \\
.069 \\
.477 \\
.458\end{array}$ & $\begin{array}{c}82(73.2) \\
28(70) \\
81(68.1) \\
82(70.1) \\
42(68.9) \\
22(71)\end{array}$ & $\begin{array}{c}30(26.8) \\
12(30) \\
38(31.9) \\
35(29.9) \\
19(31.1) \\
9(29)\end{array}$ & $\begin{array}{l}.004 * * \\
.389 \\
.342 \\
.084 \\
.404 \\
.377\end{array}$ & $\begin{array}{c}109(98.2) \\
39(100) \\
116(97.5) \\
116(99.1) \\
60(98.4) \\
29(96.7)\end{array}$ & $\begin{array}{c}2(1.8) \\
0(0) \\
3(2.5) \\
1(0.9) \\
1(1.6) \\
1(3.3)\end{array}$ & $\begin{array}{l}.581 \\
.397 \\
.517 \\
.111 \\
.631 \\
.496\end{array}$ & $\begin{array}{c}112(75.2) \\
40(26.8) \\
120(80.5) \\
118(79.2) \\
62(41.6) \\
31(20.8)\end{array}$ \\
\hline $\begin{array}{c}\text { 8. Relapse factors } \\
\text { Withdrawal symptoms } \\
\text { Stressors } \\
\text { Social problems } \\
\text { Friends } \\
\text { craving } \\
\text { Loneliness } \\
\text { Money availability } \\
\text { Sexual pleasure } \\
\text { Residence } \\
\text { Others }\end{array}$ & $\begin{array}{c}18(69.2) \\
48(69.6) \\
33(70.2) \\
30(85.7) \\
29(80.6) \\
11(68.8) \\
15(88.2) \\
10(66.7) \\
12(75) \\
40(75.5)\end{array}$ & $\begin{array}{c}8(30.8) \\
21(30.4) \\
14(29.8) \\
5(14.3) \\
7(19.4) \\
5(31.3) \\
2(11.8) \\
5(33.3) \\
4(25) \\
13(24.5)\end{array}$ & $\begin{array}{l}.241 \\
.052 * \\
.161 \\
.100 \\
.330 \\
.316 \\
.180 \\
.262 \\
.553 \\
.501\end{array}$ & $\begin{array}{c}21(80.8) \\
43(62.3) \\
32(66.7) \\
25(73.5) \\
25(69.4) \\
10(62.5) \\
14(87.5) \\
11(73.3) \\
12(75) \\
33(62.3)\end{array}$ & $\begin{array}{c}5(19.2) \\
26(37.7) \\
16(33.3) \\
9(26.5) \\
11(30.6) \\
6(37.5) \\
2(12.5) \\
4(26.7) \\
4(25) \\
20(37.7)\end{array}$ & $\begin{array}{l}.074 \\
.176 \\
.555 \\
.235 \\
.437 \\
.445 \\
.052^{*} \\
.404 \\
.335 \\
.238\end{array}$ & $\begin{array}{l}25(96.2) \\
67(97.1) \\
47(97.9) \\
34(100) \\
34(97.1) \\
15(93.8) \\
17(100) \\
14(93.3) \\
15(100) \\
51(96.2)\end{array}$ & $\begin{array}{c}1(3.8) \\
2(2.9) \\
1(2.1) \\
0(0) \\
1(2.9) \\
1(6.3) \\
0(0) \\
1(6.7) \\
0(0) \\
2(3.8)\end{array}$ & $\begin{array}{l}.442 \\
.449 \\
.695 \\
.454 \\
.558 \\
.292 \\
.692 \\
.276 \\
.724 \\
.292 \\
\end{array}$ & $\begin{array}{c}26(17.8) \\
69(47.3) \\
48(32.9) \\
35(24) \\
36(24.7) \\
16(11) \\
17(11.6) \\
15(10.3) \\
16(11) \\
53(36.3)\end{array}$ \\
\hline
\end{tabular}

$\chi^{2}$ test $* \mathrm{p} \leq .05, * * \mathrm{p}<.01$

ๆe.g., losing hope in their recovery, selling drugs themselves, to get physical energy, and be sociable 
Table 2. Mean, standard deviation and correlations between stigma, psychological distress, and the predictor variables

\begin{tabular}{|c|c|c|c|c|c|c|c|c|c|c|c|c|c|c|c|}
\hline Variables & 1 & 2 & 3 & 4 & 5 & 6 & 7 & 8 & 9 & 10 & 11 & 12 & 13 & 14 & 15 \\
\hline 1. Psychological distress & -- & & & & & & & & & & & & & & \\
\hline 2. Depression & $.802 * *$ & -- & & & & & & & & & & & & & \\
\hline 3. Anxiety & $.807 * *$ & $.473 * *$ & -- & & & & & & & & & & & & \\
\hline 4. Stress & $.825 * *$ & $.573 * *$ & $.636^{* *}$ & -- & & & & & & & & & & & \\
\hline 5. SSAD Awareness & -.007 & .086 & -.017 & -.060 & -- & & & & & & & & & & \\
\hline 6. SSAD Agreement & .143 & $.183^{*}$ & .096 & .131 & $.459 * *$ & -- & & & & & & & & & \\
\hline 7. SSAD Self-occurrence & $.417 * *$ & $.445^{* *}$ & $.322 * *$ & $.385^{* *}$ & .018 & .114 & -- & & & & & & & & \\
\hline 8. SSAD Shame & $.462 * *$ & $.482 * *$ & $.347 * *$ & $.385^{* *}$ & $-.025-$ & .027 & $.735^{* *}$ & -- & & & & & & & \\
\hline 9. Age (years) & -.127 & -.117 & -.065 & -.116 & .021 & -.029 & $-.176^{*}$ & -.128 & -- & & & & & & \\
\hline 10. Chronicity (years) & $-.196 *$ & $-.224 * *$ & -.086 & $-.214 * *$ & .090 & -.040 & $-.202 *$ & $-.173 *$ & $.613 * *$ & -- & & & & & \\
\hline 11. No of quitting trials & -.063 & -.105 & .000 & -.017 & .059 & $.062-$ & .016 & .078 & $.214^{* *}$ & $.331 * *$ & -- & & & & \\
\hline 12. No of hospital admissions & .123 & .078 & .120 & .155 & .068 & -.027 & .157 & $.241 * *$ & .136 & $.256 * *$ & $.459 * *$ & -- & & & \\
\hline 13. Length of hospital stay (days) & $-.265 * *$ & $-.325 * *$ & -.074 & -.090 & -.066 & -.032 & .017 & -.116 & .059 & .119 & .127 & .018 & -- & & \\
\hline 14. No of abused drugs & $-.183^{*}$ & -.145 & -.146 & -.131 & $.187^{*}$ & .105 & -.056 & -.009 & .100 & $.309 *$ & $.282 * *$ & $.222 * *$ & $.218 * *$ & -- & \\
\hline 15. No of relapse factors & -.016 & .043 & -.033 & -.029 & .134 & .083 & .042 & .119 & -.023 & $.163 *$ & .087 & $.207^{*}$ & .003 & $.288 * *$ & -- \\
\hline M & 27 & 9.2 & 7.8 & 10 & 64.3 & 61.7 & 43.9 & 40.8 & 32.5 & 14.9 & 14.2 & 4.1 & 37.1 & 3.2 & 2.2 \\
\hline SD & 13 & 5.4 & 5.6 & 4.2 & 8.4 & 10.1 & 11 & 11.8 & 6.8 & 9 & 21 & 5 & 49.2 & 1.6 & 1.2 \\
\hline
\end{tabular}

$\mathrm{SSAD}=$ Self-Stigma in Alcohol Dependence Scale; $\boldsymbol{\uparrow}=$ variables not transformed; $\mathrm{M}=$ mean; $\mathrm{SD}=$ standard deviation., ${ }^{*} \mathrm{p} \leq .05, * * \mathrm{p}<.01$

Table 3. Hierarchical multiple regression predicting stigma variables, overall psychological distress, depression, anxiety, and stress (SSAD = Self-Stigma in Alcohol Dependence Scale; $r$

$=$ Pearson's correlation; $\beta=$ Beta standardized coefficients; $\mathrm{SE}=$ Standard error; $95 \% \mathrm{CI}=95 \%$ Confidence interval for $\beta$

${ }^{\text {a }}$ Parameters adjusted for age and gender, ${ }^{*} \mathrm{p} \leq .05,{ }^{* *} \mathrm{p}<.01,{ }^{* * *} \mathrm{p}<.001$ )

\begin{tabular}{|c|c|c|c|c|c|c|c|c|}
\hline Criterion & Predictors $^{a}$ & $\mathbf{r}$ & $\beta$ & Adjusted R $\mathbf{R}^{2}$ & $\mathbf{R}^{2}$ change ${ }^{a}$ & F Change & SE & $95 \%$ CI for $\rho$ \\
\hline \multirow[b]{2}{*}{ SSAD Awareness } & & & & .045 & .075 & $3.864 *$ & .061 & \\
\hline & $\begin{array}{c}\text { Alcohol } \\
\text { Bango } \\
\text { No of abused drugs }\end{array}$ & $\begin{array}{l}.249^{* *} \\
.216^{* *} \\
.187^{*}\end{array}$ & $\begin{array}{l}.223 * \\
.149 \\
-.065\end{array}$ & & & & & $\begin{array}{l}.000, .056 \\
-.008, .050 \\
-.079, .046\end{array}$ \\
\hline \multirow{2}{*}{ SSAD Agreement } & & & & .048 & .065 & $10.165^{* *}$ & .081 & \\
\hline & Bango & $.251 * *$ & $.258^{* *}$ & & & & & $.018, .079$ \\
\hline \multirow{2}{*}{ SSAD Self-occurrence } & & & & .039 & .012 & 1.846 & 10.79 & \\
\hline & Chronicity & $-.202 *$ & -.133 & & & & & $-.399, .074$ \\
\hline \multirow[b]{2}{*}{ SSAD Shame } & & & & .061 & .042 & $3.310^{*}$ & 11.32 & \\
\hline & $\begin{array}{c}\text { Chronicity } \\
\text { No of hospital admis- } \\
\text { sions }\end{array}$ & $\begin{array}{l}-.173 * \\
.241 * *\end{array}$ & $\begin{array}{l}-.180 \\
.152\end{array}$ & & & & & $\begin{array}{l}-.482, .015 \\
-.020, .735\end{array}$ \\
\hline \multirow[t]{7}{*}{ Psychological distress } & & & & .34 & .31 & $11.5^{* * *}$ & .21 & \\
\hline & Chronicity & $-.196^{*}$ & .139 & & & & & $-.268,-.055$ \\
\hline & $\begin{array}{l}\text { Number of abused } \\
\text { drugs }\end{array}$ & $-.183^{*}$ & -.189 & & & & & $-.060, .340$ \\
\hline & Cannabis & $-.177^{*}$ & .09 & & & & & $-.07, .176$ \\
\hline & Length of hospital stay & $-.265 * *$ & $-.186 * *$ & & & & & $-.243,-.036$ \\
\hline & SSAD self-occurrence & $.417 * *$ & .183 & & & & & $0, .009$ \\
\hline & SSAD Shame & $.462 * *$ & $.374 * * *$ & & & & & $.004, .012$ \\
\hline \multirow[t]{7}{*}{ Depression } & & & & .39 & .36 & $14.5^{* * *}$ & .25 & \\
\hline & Chronicity & $-.224 *$ & .028 & & & & & $-.191, .26$ \\
\hline & Cannabis & $-.177^{*}$ & -.045 & & & & & $-.130, .065$ \\
\hline & Length of hospital stay & $-.325 * *$ & $-.259 * * *$ & & & & & $-.36,-.118$ \\
\hline & SSAD Agreement & $.183^{*}$ & $.156^{*}$ & & & & & $.096,1.073$ \\
\hline & SSAD self-occurrence & $.445^{* *}$ & .175 & & & & & $-.001, .01$ \\
\hline & SSAD Shame & $.482 * *$ & $.359 * * *$ & & & & & $.004, .015$ \\
\hline \multirow[t]{4}{*}{ Anxiety } & & & & .18 & .17 & $10.1 * * *$ & .3 & \\
\hline & $\begin{array}{l}\text { Relapse out of loneli- } \\
\text { ness }\end{array}$ & $.178^{*}$ & $.152^{*}$ & & & & & $.003, .325$ \\
\hline & SSAD self-occurrence & $.322 * *$ & .157 & & & & & $-.002, .011$ \\
\hline & SSAD Shame & $.347^{* *}$ & $.243^{*}$ & & & & & $.001, .013$ \\
\hline \multirow[t]{5}{*}{ Stress } & & & & .25 & .20 & $10 * * *$ & 3.5 & \\
\hline & Chronicity & $-.214 * *$ & .013 & & & & & $-3.029,3.438$ \\
\hline & Job & $-.189 *$ & -.046 & & & & & $-1.825, .979$ \\
\hline & SSAD self-occurrence & $.385^{* *}$ & $.213^{*}$ & & & & & $.002, .157$ \\
\hline & SSAD Shame & $.385^{* *}$ & $.274 *$ & & & & & $.023, .170$ \\
\hline
\end{tabular}


Table 4. Additional material, Mean, SD, t-test for SSAD subscales mean item scores

\begin{tabular}{|c|c|c|c|c|c|}
\hline & $\mathbf{M}$ & $S D$ & $\mathbf{t}$ & $\mathbf{d f}$ & $\mathbf{9 5 \%} \mathbf{C I}$ \\
\hline $\begin{array}{c}\text { SSAD Aware- } \\
\text { ness }\end{array}$ & 4.01 & .54 & $22.925^{* * *}$ & 146 & $.925,1.100$ \\
\hline $\begin{array}{c}\text { SSAD Agree- } \\
\text { ment }\end{array}$ & 3.85 & .63 & $16.382^{* * *}$ & 146 & $.749, .955$ \\
\hline $\begin{array}{c}\text { SSAD Self- } \\
\text { occurrence }\end{array}$ & 2.75 & .69 & $4.331^{* * *}$ & 146 & $.360, .135$ \\
\hline SSAD Shame & 2.56 & .73 & $7.366^{* * *}$ & 147 & $.562, .324$ \\
\hline
\end{tabular}

Table 5. For the first 3 subscales scores $\mathrm{N}=147$ while for the fourth subscale $\mathrm{N}=148$ participants

\begin{tabular}{l|l|l|l|l|l|l|}
\hline \multirow{3}{*}{ Variables } & \multicolumn{2}{|c|}{ Depression } & \multicolumn{2}{c|}{ Anxiety } & \multicolumn{2}{c|}{ Stress } \\
\cline { 2 - 7 } & $\begin{array}{c}14<\text { Low } \\
(\mathrm{N}=113)\end{array}$ & $\begin{array}{c}14 \geq \text { High } \\
(\mathrm{N}=35)\end{array}$ & $\begin{array}{c}10<\text { Low } \\
(\mathrm{N}=99)\end{array}$ & $\begin{array}{c}10 \geq \text { High } \\
(\mathrm{N}=49)\end{array}$ & $\begin{array}{c}19<\text { Low } \\
(\mathrm{N}=145)\end{array}$ & $\begin{array}{c}19 \geq \text { High } \\
(\mathrm{N}=3)\end{array}$ \\
$* * * \mathrm{p}=.000$
\end{tabular}

stay, and cannabis use No significant correlations were found between psychological distress or any of its dimensions with education, marital status, and income (data not shown); stress was negatively correlated with the employment status ( $r=-0.189)$, and positively correlated with the housing status $(\mathrm{r}=0.186), p$ values $<0.05$.

To test the hypotheses of this study, regressions adjusted for age and gender were conducted to examine whether use of alcohol and Bango, the number of abused drugs, disease chronicity, and numbers of hospital admissions can predict stigma Age and gender in the first step explained $0.2 \%, 0.2 \%, 4.6 \%$, and $4.4 \%$ of the variances in stereotype awareness, agreement, self-occurrence, and shame, respectively Age had a significant contribution to the variance in self-occurrence and shame $(\boldsymbol{\beta}=-0.206$ and $-0.186, p$ values $<0.05)$ (data not shown) Regression adjusted for age and gender is shown in Table 3 In the second step, alcohol consumption significantly predicted stereotype awareness $(\boldsymbol{\beta}=0.223, p<0.05)$ whereas use of Bango significantly predicted stereotype agreement $(\boldsymbol{\beta}=0.258, p<0.01)$.

Adjusted multiple regression was conducted to examine stigma, relapse variables, and the length of hospital stay as predictors of psychological distress, depression, anxiety, and stress Age and gender in the first step explained $4.9 \%, 4.3 \%, 2.4 \%$, and $6.9 \%$ of the variance in psychological distress, depression, anxiety and stress, respectively Gender had the highest contribution $(\boldsymbol{\beta}=0.220,0.214,0.188$, and 0.268$)$ (data not shown) In the second step, shame and the length of hospital stay significantly predicted psychological distress $\Delta F(6,140)=11.5$, $p<0.000, \Delta R^{2}=0.31$ Meanwhile, stereotypes agreement, shame, and the length of hospital stay significantly predicted depression $\Delta F(6,140)=14.5, p<0.000, \Delta R^{2}=0.36$ Both shame and relapse because of loneliness significantly predicted anxiety $\Delta F(3,143)=10.1, p<0.000$, $\Delta R^{2}=0.17$ whereas stereotypes self-occurrence and shame significantly predicted stress $\Delta F(4,142)=10, \mathrm{p}<0.000, \Delta R^{2}=0.20$ Examination of individual predictors indicated that shame had the highest contribution in all models $(\boldsymbol{\beta}=0.374,0.359,0.243$, and 0.274 , respectively).

\section{Discussion}

To the present moment, this is the first study that assessed stigma and psychological distress and their associated factors among Arab SUDs patients The participants endorsed high perceived stigma and to a lesser extent internalized stigma, and both stigma constructs were weakly related, which is consistent with earlier studies $[7,10,17,19,20]$ Contrary to prediction, in adjusted analysis only age and consumption of alcohol and Bango predicted stigma In addition, shame was a strong predictor of psychological distress while among disease chronicity, abused substances, relapse, sociodemographic characteristics, only short hospital stay significantly predicted distress and depressive symptoms.

Although several studies addressed the relationship between stigma and the sociodemographic variables, results are inconsistent For instance, stigma was associated with high levels of education in Vietnamese drug users who received methadone maintenance treatment [21] On the contrary, it was associated with low levels of education in American alcohol users [19] In this study, young age predicted both stereotypes self-occurrence and shame, which is in accord with an evidence denoting high prevalence of negative attitudes among younger age groups that decrease with increasing age [35] This finding entails that younger drug users are in a greater need for self-concept enhancement treatments since the damages of stigma to mental health can contribute to their continued use and relapse.

The current findings indicate that use of Bango and alcohol predicted higher perceived stigma; participants of this study, however, were multiple drug users Research documents severe cognitive and behavioral impairments (e.g hallucination) with the use of Bango [5], which participants could have personally endured or witnessed among their peers Furthermore, the stigma perceptions held by the participants could be a mere reflection of the negative attitudes that the general Egyptian public hold about alcohol and other substances prohibited by Islam [36,37] In Egypt alcohol use, for religious reasons, is considered a sin [38]; the common slang "Khamorgy" is used to humiliate an alcohol drinker

The poor association observed between perceived and internalized stigma supports claims of a former developmental study that both constructs are distinct [11] Other factors (e.g experiences of rejection) may play roles in the self-devaluation process.

The current results indicate that both perceived and internalized stigma can have a detrimental effect on psychological health Although stigma perceptions are not self-relevant, stereotypes agreement significantly predicted depressive symptoms Moreover, internalized stigma (specifically, shame) was a meaningful construct that contributed to psychological distress, depression, anxiety, and stress symptoms These results are congruent with reports from earlier studies that also included experiences of rejection as a dimension of self-stigma [16,20,21,25] Accordingly, the present study suggests that the role of stigma triggering negative self-feelings such as shame can be the most destructive aspect of stigma, regardless of encountering rejection Therefore, shame should be the target of interventions that aim to restructure resiliency and combat effects of internalized stigma on mental well-being in this disadvantaged population.

Contrary to expectations, chronicity of SUDs, larger numbers of used substances, use of heroin and cannabis (known to induce depressive symptoms) could not predict psychological distress The literature highlights a dose-related effect of heroin [39], cannabis, and marijuana on mental health in regular users [40,41] However, the present study did not examine routes of administration, doses, and frequencies of substance use while participants reported all the drugs that they used in their lifetime-they possibly shifted between many substances and eventually were dependent on one or two drugs Besides, most participants used several substances simultaneously, which probably weakened the estimated associations between distress outcomes and use of specific single substances.

Shorter hospital stays, indicative of recent hospital admission, significantly predicted higher psychological distress and depressive symptoms; most participants reported receiving a dose on the day 
of admission or the day before This finding lends further support to the contribution of current and recent substance use to psychological distress and depressive symptoms-which is in line with outcomes of similar studies that recorded an association of depression with fewer total days of treatment [39] and active drug use [25]

Among all relapse factors, only loneliness significantly predicted anxiety symptoms The reason why loneliness in particular predicted anxiety is unclear Loneliness was fairly reported while social problems and stressors were highly stated as relapse factors Meanwhile, most participants (as usual in Egypt) were staying with their families, and stress symptoms were associated with the housing status-these results might suggest that participants felt unsupported and/or lonely though staying with their families Evidence denotes a need for acceptance among anxious people who also feel lonely [42], which highlights a likelihood that anxious participants could have encountered rejection within their close social networks This claim draws support from the strong association between family functioning and psychological distress among SUDs patients [2] Future studies should address the effect of social support and rejection on the mental health of SUDs victims.

\section{Limitations}

This study has several weaknesses, which must be acknowledged Generalizability of the findings is limited since participants were not representative of the Egyptian SUDs population: under-educated, lowincome, multiple-substance users, prominently men recruited from a government hospital Besides, data were prone to self-report bias, and the sample was relatively small because of logistic reasons Additionally, the scale that measured stigma (SSAD) ignores experiences of rejection and stigma coping, which indicates underestimation of stigma in this study.

\section{Conclusion}

The study at hand suggests that stigma (the shame component) has the highest contribution to psychological distress, depression, anxiety, and stress Findings necessitate the use of special shame management interventions to improve psychological health, especially among young users of illicit drugs This study suggests a need to investigate experiences of rejection, social support, and quality of life in larger samples to broaden understanding of stigma and its effect on psychological wellbeing among Egyptian SUDs patients

\section{Conflicts of interest}

The author reports no conflict of interest to declare.

\section{References}

1. UNODC, World Drug Report. (United Nations publication, Sales No. E.16.XI.7). Retrieved May 9, 2017, from https://www.unodc.org/doc/wdr2016/WORLD_DRUG REPORT_2016_web.pdf. 2016.

2. Gyawali B (2016) Prevalence and correlates of psychological distress symptoms among patients with substance use disorders in drug rehabilitation centers in urban Nepal: A cross-sectional study. BMC Psychiatry 16: 314

3. Sweileh WM (2014) Substance use disorders in Arab countries: research activity and bibliometric analysis. Subst Abuse Treat Prev Policy 9: 33.

4. Hamdi E (2016) Sociodemographic indicators for substance use and abuse in Egypt. J Addict Pre 4: 8.

5. Dawood AE-WA (2009) Risk factors lead to Bango abuse among drivers and secondery school students in Assiut province. Iranian Journal of Toxicology 2: 1-4

6. Bassiony MM, Youssef UM, Hassan MS, Salah El-Deen GM, El-Gohari H, et al. (2017) Cognitive Impairment and Tramadol Dependence. J Clin Psychopharmacol 37: 61-66. [Crossref]
7. Ahern J, Stuber J, Galea S (2007) Stigma, discrimination and the health of illicit drug users. Drug Alcohol Depend 88: 188-196. [Crossref]

8. Schomerus G (2010) The stigma of alcohol dependence compared with other mental disorders: a review of population studies. Alcohol Alcohol 46: $105-112$

9. Luo T (2014) Stigmatization of people with drug dependence in China: A communitybased study in Hunan province. Drug Alcohol Depend 134: 285-289.

10. Rivera AV (2014) Internalized stigma and sterile syringe use among people who inject drugs in New York City, 2010-2012. Drug Alcohol Depend 1: 259-264.

11. Luoma JB (2013) Self-stigma in substance abuse: Development of a new measure. $J$ Psychopathol Behav Assess 35: 223-234.

12. Schomerus G (2017) Public attitudes towards alcohol dependence. Psychiatr Prax 2010. 37(3):Abstract. Retrieved April, 2017, from http://www.thieme-connect.com/ DOI/DOI?10.1055/s-0029-1223438

13. Link BG and Phelan JC (2001) Conceptualizing Stigma. Annu Rev Sociol 27: 363-385.

14. Schomerus G (2017) Self-stigma in alcohol dependence: Consequences for drinkingrefusal self-efficacy. Drug Alcohol Depend 114: 12-17.

15. Corrigan PW, Larson JE and RUSch (2009) Self-stigma and the "why try" effect: impact on life goals and evidence-based practices. World Psychiatry 8: 75-81.

16. Cama E (2016) Internalized stigma among people who inject drugs. Subst Use Misuse 51: 1664-1668.

17. Semple SJ, Grant I and Patterson TL (2005) Utilization of drug treatment programs by methamphetamine users: The role of social stigma. Am J Addict 14: 367-380.

18. Szafranski DD (2017) Integrated, exposure-based treatment for PTSD and comorbid substance use disorders: Predictors of treatment dropout. Addict Behav 73: 30-35.

19. Keyes KM, Hatzenbuehler ML, McLaughlin KA, Link B, Olfson M, et al. (2010) Stigma and treatment for alcohol disorders in the United States. Am J Epidemiol 172: 1364-1372. [Crossref]

20. Luoma JB (2007) An investigation of stigma in individuals receiving treatment for substance abuse. Addict Behav 32: 1331-1346.

21. Tran BX (2016) Drug addiction stigma in relation to methadone maintenance treatment by different service delivery models in Vietnam. BMC Public Health 16(238).

22. Ali AM (2019) Factor structure of the depression anxiety stress Scale-21 (DASS-21): Unidimensionality of the Arabic version among Egyptian drug users. Substance Abuse Treatment, Prevention, and Policy 14: 40.

23. Hobbs JDJ (2011) Meta-analysis of supplemental treatment for depressive and anxiety disorders in patients being treated for alcohol dependence. Am J Addict 20: 319-329.

24. Green KM (2012) Interrelationship of substance use and psychological distress over the life course among a cohort of urban African Americans. Drug Alcohol Depend 123 : 239-248.

25. Latkin C (2012) The relationship between drug user stigma and depression among inner-city drug users in Baltimore, MD. J Urban Health 90: 147-157.

26. Sinha R (2008) Chronic stress, drug use, and vulnerability to addiction. Ann N Y Acad Sci 1141: 105-130. [Crossref]

27. Richman LS and Lattanner MR (2014) Self-regulatory processes underlying structural stigma and health. Soc Sci Med 103: 94-100.

28. Coombs RH (2004) Handbook of addictive disorders: A practical guide to diagnosis and treatment. John Wiley \& Sons, 2004. P 82. Retrieved from https://books.google. co.jp/books?id=sO7S8UpXhjAC\&dq=thought + process + rumination + and + shame\&hl= ar\&source=gbs_navlinks_s.

29. Luoma JB (2017) Slow and steady wins the race: A randomized clinical trial of acceptance and commitment therapy targeting shame in substance use disorders. $J$ Consult Clin Psychol 80: 43-53.

30. Ali B (2017) Distress tolerance interacts with circumstances, motivation, and readiness to predict substance abuse treatment retention. Addict Behav 73: 99-104.

31. Bravo AJ and Pearson MR (2017) In the process of drinking to cope among college students: An examination of specific vs. global coping motives for depression and anxiety symptoms. Addict Behav 73: 94-98.

32. Reid P (2012) Comorbidity between psychological distress and drug use among patients in treatment centres in Jamaica: implications for policies and programme design. Texto Contexto Enferm, Florianópolis 21: 133-140

33. Lovibond PF and SH (1995) Lovibond, Manual for the Depression Anxiety Stress Scales (2nd ed.). Psychology Foundation, Sydney. 
34. Tran TD (2013) Validation of the depression anxiety stress scales (DASS) 21 as a screening instrument for depression and anxiety in a rural community-based cohort of northern Vietnamese women. BMC Psychiatry 13: 24-24.

35. Adlaf EM, Hamilton HA, Wu F, Noh S (2009) Adolescent stigma towards drug addiction: effects of age and drug use behaviour. Addict Behav 34: 360-364. [Crossref]

36. Jamal AA and Milner HV (2013) Economic and cultural sources of preferences for globalization in Egypt. American political science meetings, Chicago, Ill. Retrieved from: https://scholar.princeton.edu/sites/default/files/hvmilner/files/jamalmilner_ apsa_2013.pdf, 2013.

37. Mostafa MM (2011) An investigation of Egyptian consumers' attitudes toward ethical issues in advertising. Journal of Promotion Management 17: 42-60.
38. Negm MG (2014) Prevalence of substance abuse among adolescent school students in Zagazig. Egypt J Psychiatry 35: 161-166.

39. Havard A (2006) Depression among heroin users: 12-Month outcomes from the Australian Treatment Outcome Study (ATOS). J Subst Abuse Treat 30: 355-362.

40. Bolla KI, Brown K, Eldreth D, Tate K, Cadet JL (2002) Dose-related neurocognitive effects of marijuana use. Neurology 59: 1337-1343. [Crossref]

41. Johns A (2001) Psychiatric effects of cannabis. Br J Psychiatry 178: 116-122. [Crossref]

42. Ginter EJ (1996) Loneliness, perceived social support, and anxiety among Israel adolescents. Psychol Rep 79: 335-341.

Copyright: $@ 2019$ Ali AM. This is an open-access article distributed under the terms of the Creative Commons Attribution License, which permits unrestricted use, distribution, and reproduction in any medium, provided the original author and source are credited. 